\title{
Influence of manipulating hypoxia in solid tumors on the radiation dose-rate effect in vivo, with reference to that in the quiescent cell population.
}

\section{AUTHOR(S):}

Masunaga, Shin-ichiro; Hirayama, Ryoichi; Uzawa, Akiko; Kashino, Genro; Takata, Takushi; Tanaka, Hiroki; Suzuki, Minoru; ... Koike, Sachiko; Ando, Koichi; Ono, Koji

\section{CITATION:}

Masunaga, Shin-ichiro ...[et al]. Influence of manipulating hypoxia in solid tumors on the radiation dose-rate effect in vivo, with reference to that in the quiescent cell population. Japanese journal of radiology 2010, 28(2): 132-142

\section{ISSUE DATE:}

2010-02

URL:

http://hdl.handle.net/2433/128868

\section{RIGHT:}

The original publication is available at www.springerlink.com; This is not the published version. Please cite only the published version.; この 論文は出版社版でありません。引用の際には出版社版をご確認ご利用 ください。 


\section{Editorial Manager(tm) for Japanese Journal of Radiology} Manuscript Draft

Manuscript Number: RMED-273R3

Title: Influence of manipulating hypoxia in solid tumors on radiation dose-rate effect in vivo, referring to that in quiescent cell population

Article Type: Original Article

Keywords: Dose-rate effect; Manipulating hypoxia; Quiescent cell; Carbon-ion beams; Gamma-rays

Corresponding Author: Dr. Shin-ichiro Masunaga, M.D., Ph.D.

Corresponding Author's Institution: Research Reactor Institute, Kyoto University

First Author: Shin-ichiro Masunaga, M.D., Ph.D.

Order of Authors: Shin-ichiro Masunaga, M.D., Ph.D.; Ryoichi Hirayama, Ph.D.; Akiko Uzawa, Ph.D.; Genro Kashino, Ph.D.; Takushi Takata, Ph.D.; Hiroki Tanaka, Ph.D.; Minoru Suzuki, M.D., Ph.D.; Yuko Kinashi, M.D., Ph.D.; Yong Liu, M.D., Ph.D.; Sachiko Koike, Ph.D.; Koichi Ando, Ph.D.; Koji Ono, M.D., Ph.D. 
Influence of manipulating hypoxia in solid tumors on radiation dose-rate effect in vivo, referring to that in quiescent cell population

Shin-ichiro Masunaga ${ }^{1}$, Ryoichi Hirayama ${ }^{2}$, Akiko Uzawa ${ }^{2}$, Genro Kashino ${ }^{1}$, Takushi Takata ${ }^{3}$, Hiroki Tanaka ${ }^{4}$, Minoru Suzuki ${ }^{1}$, Yuko Kinashi ${ }^{5}$, Yong Liu ${ }^{1}$, Sachiko Koike", Koichi Ando ${ }^{6}$ and Koji Ono ${ }^{1}$

${ }^{1}$ Particle Radiation Oncology Research Center, ${ }^{4}$ Radiation Medical Physics and ${ }^{5}$ Radiation Safety and Control, Research Reactor Institute, Kyoto University, Kumatori, Osaka, Japan.

${ }^{2}$ Heavy-Ion Radiobiology Research Group, Research Center for Charged Particle Therapy, National Institute of Radiological Sciences, Chiba, Japan.

3 Wakasawan Energy Research Center, Tsuruga, Fukui, Japan. ${ }^{6}$ Heavy Ion Medical Center, Gunma University, Gunma, Japan.

\section{Running head:}

Influence of manipulating hypoxia on radiation dose-rate effect

\section{All correspondence to:}

Shin-ichiro Masunaga, M.D., Ph.D.

Particle Radiation Oncology Research Center

Research Reactor Institute, Kyoto University

2-1010, Asashiro-nishi, Kumatori-cho,

Sennan-gun, Osaka 590-0494, Japan.

Tel: +81-72-451-2406, 2487, Fax: +81-72-451-2627

E-mail: smasunaerri.kyoto-u.ac.jp

\section{Acknowledgments}

This study was supported, in part, by a Grant-in-aid for Scientific Research (C) (20591493) from the Japan Society for the Promotion of Science.

\section{Original article}

The authors declare no conflicts of interest concerning this study. 
Abstract

Purpose: To clarify the effect of manipulating intratumor hypoxia on radiosensitivity under reduced dose-rate (RDR) irradiation.

Methods: Tumor-bearing mice were continuously given

5-bromo-2'-deoxyuridine (BrdU) to label all proliferating (P) cells. They received $\gamma$-rays or accelerated carbon-ion beams at high dose-rate (HDR) or RDR with or without tumor clamping to induce hypoxia. Some mice without clamping received nicotinamide, an acute hypoxia-releasing agent or misonidazole, a hypoxic cell radio-sensitizer before irradiation. The responses of quiescent (Q) and total (= P+Q) cells were assessed by the micronucleus frequency using immunofluorescence staining for BrdU.

Results: The clearer decrease in radiosensitivity in $Q$ than total cells after RDR $\gamma$-ray irradiation was suppressed with carbon-ion beams, especially with a higher linear energy transfer value. Repressing the decrease in the radiosensitivity under RDR irradiation through keeping tumors hypoxic during irradiation and enhancing the decrease in the radiosensitivity by nicotinamide were clearer with $\gamma$-rays and in total cells than with carbon-ion beams and in Q cells, respectively. Inhibiting the decrease in the radiosensitivity by misonidazole was clearer with $\gamma$-rays and in $Q$ cells than with carbon-ion beams and in total cells, respectively.

Conclusion: Manipulating hypoxia during RDR as well as HDR irradiation influences tumor radiosensitivity, especially with $\gamma$-rays.

\section{Key words :}

Dose-rate effect; Manipulating hypoxia; Quiescent cell; Carbon-ion beams; $\gamma$-Rays 
Influence of manipulating hypoxia on radiation dose-rate effect/Page 2

\section{Introduction}

Intensity modulated radiotherapy (IMRT) and stereotactic irradiation have become common as a new radiotherapy technique for treatment of malignancies. Both modalities generally use multiple arc or fixed-portal radiation beams, and radiation beams are exposed intermittently. These techniques often require $30 \mathrm{~min}$ or a longer time in one treatment session for precise positioning of patients. ${ }^{1,2}$ Prolongation of irradiation time may reduce a radiation effect, and evokes a major concern for the dose rate effect. Thus, it is needed to clarify the effect of the reduction of dose rate on the radiosensitivity of tumors in vivo.

When using low linear energy transfer (LET) radiation, lowering the dose rate is thought to reduce late effects in normal tissue much more than it decreases tumor control. Thus, the "therapeutic ratio" increases as the dose rate decreases, because the therapeutic ratio is equal to the ratio of tumor control to normal tissue complications. Further, the difference between early and late effects for low dose-rate radiotherapy, as well as improving the therapeutic ratio, allows complete treatment in a short period of time, minimizing the effects of tumor repopulation. In other words, decreasing the dose rate increases the therapeutic ratio, limited only by tumor cell repopulation. ${ }^{3}$ This is the primary rationale for low dose-rate radiotherapy using low LET radiation. High LET radiation is more effective than low LET X- or $\gamma$-radiation at inducing biologic damage. High LET radiation results in a greater relative biological effectiveness (RBE) value for cell killing, a reduced oxygen effect, and a reduced dependence on the cell cycle and the irradiation dose 
Influence of manipulating hypoxia on radiation dose-rate effect/Page 3

rate. ${ }^{4}$

Manipulating hypoxia in solid tumors during irradiation apparently influences tumor radiosensitivity under high dose-rate (HDR) irradiation using low LET radiation. ${ }^{5}$ However, its significance in solid tumors irradiated at a reduced dose rate (RDR) is less clear in vivo, whether low or high LET radiation is employed.

Many cells in solid tumors are quiescent in situ but still clonogenic. ${ }^{6}$ The quiescent (Q) tumor cells are more resistant to low-LET radiation because of their larger hypoxic fraction and greater capacity to recover from potentially lethal damage (PLD) than proliferating (P) tumor cells. The rationale for low dose-rate radiotherapy does not take into account the response of $Q$ tumor cells at all.

Thus, in this study, we tried to elucidate the effect of manipulating hypoxia in irradiated solid tumors at a RDR with low-LET $\gamma$-rays or high-LET $290 \mathrm{MeV} / \mathrm{u}$ accelerated carbon-ion beams in vivo, compared with HDR irradiation. Further, the responses of the total (= $P+Q)$ and $Q$ cell populations in irradiated solid tumors were separately detected with the method for selectively detecting the response of $Q$ cells within solid tumors. ${ }^{7}$ This is the first attempt to clarify the direct relationship between the irradiation dose rate effect and the oxygen effect in vivo, referring to the response of the $Q$ cell population in irradiated solid tumors. 
Influence of manipulating hypoxia on radiation dose-rate effect/Page 4

\section{Materials and Methods}

\section{Mice and tumors}

SCC VII squamous cell carcinoma cell line derived from C3H/He mice was maintained in vitro in Eagle's minimum essential medium supplemented with 12.5 fetal bovine serum. The tumor cells (1.0 x $10^{5}$ ) were inoculated subcutaneously into the left hind leg of 9-week-old syngeneic female C3H/He mice (Japan Animal Co., Ltd., Osaka, Japan). Fourteen days later, the tumors, approximately $1 \mathrm{~cm}$ in diameter, were employed for experimental treatment, and the body weight of the tumor-bearing mice was 22.1 \pm 2.3 (Mean \pm SD) 9. Mice were handled according to the Recommendations for Handling of Laboratory Animals for Biomedical Research, compiled by the Committee on Safety Handling Regulations for Laboratory Animal Experiments. Incidentally, the p53 of SCC VII tumor cells is the wild type.

\section{Labeling with 5-bromo-2'-deoxyuridine (BrdU)}

Nine days after the inoculation, mini-osmotic pumps (Durect Corporation, Cupertino, CA) containing BrdU dissolved in physiological saline (250 mg/ml) were implanted subcutaneously to label all $\mathrm{P}$ cells for 5 days. The percentage of labeled cells after continuous labeling with BrdU was $55.3 \pm 4.5 \%$, and reached a plateau at this stage. Therefore, tumor cells not incorporating BrdU after continuous labeling were regarded as Q cells.

\section{Treatment}

After the labeling with BrdU, tumor-bearing mice received $\gamma$-ray or accelerated carbon-ion whole-body irradiation, with the animal held in a specially designed device made of acrylic resin with the tail or all four legs firmly fixed with adhesive tape with no anesthetic. Some tumors were made totally hypoxic by clamping the proximal end $15 \mathrm{~min}$ 
Influence of manipulating hypoxia on radiation dose-rate effect/Page 5

before irradiation. 7 This clamping for 15 min did not influence clonogenic cell survival or the level of micronucleation.

$\gamma$-Rays were delivered with a cobalt-60 $\gamma$-ray irradiator at a dose rate of 2.5 or $0.039 \mathrm{~Gy} / \mathrm{min}$.

Carbon-12 ions were accelerated up to $290 \mathrm{MeV} / \mathrm{u}$ by the synchrotron of the Heavy Ion Medical Accelerator installed at National Institute of Radiological Sciences in Chiba, Japan. The dose rate was regulated through a beam attenuation system, and irradiation was conducted using horizontal carbon beams with a dose rate of 1.0 or $0.035 \mathrm{~Gy} / \mathrm{min}$. The LET of the carbon ion beam with the 6-cm spread-out Bragg peak (SOBP) ranges from $14 \mathrm{keV} / \mu \mathrm{m}$ to greater than $200 \mathrm{keV} / \mu \mathrm{m}$, depending on depth. A desired LET beam was obtained by selecting the depth along the beam path using a Lucite range shifter. An LET of 18 and $50 \mathrm{keV} / \mu \mathrm{m}$ at the middle of the plateau and the SOBP were employed here, respectively. ${ }^{8}$

Irradiated tumor-bearing mice were divided into 4 groups. I) Tumors were excised immediately after irradiation only under aerated conditions. II) Tumors were kept totally hypoxic during irradiation, then excised immediately after irradiation. III) The tumor-bearing mice received an intraperitoneal administration of an acute hypoxia-releasing agent, nicotinamide (1000 mg/kg of mouse weight) dissolved in physiological saline 60 min before irradiation, then the tumors were excised immediately after irradiation under aerated conditions. IV) The tumor-bearing mice received an intraperitoneal administration of a hypoxic cell radio-sensitizer, misonidazole (1000 mg/kg of mouse weight) dissolved in physiological saline 30 min before irradiation, then the tumors were excised immediately after irradiation under aerated conditions. All the doses employed, sequences and timing for nicotinamide, misonidazole and irradiation were appropriate enough 
Influence of manipulating hypoxia on radiation dose-rate effect/Page 6

to function completel $\mathrm{y}^{9,10}$.

Each treatment group also included mice not pretreated with BrdU.

\section{Immunofluorescence staining of BrdU-labeled cells and micronucleus (MN) assay}

Tumors excised from the mice given BrdU were minced and trypsinized [0.05\% trypsin and 0.02\% ethylenediamine-tetraacetic acid (EDTA) in phosphate-buffered saline (PBS), $\left.37^{\circ} \mathrm{C}, 20 \mathrm{~min}\right]$. Tumor cell suspensions were incubated for $72 \mathrm{~h}$ in tissue culture dishes containing complete medium and $1.0 \mu \mathrm{g} / \mathrm{ml}$ of cytochalasin-B to inhibit cytokinesis while allowing nuclear division, and the cultures were then trypsinized and cell suspensions were fixed. After the centrifugation of fixed cell suspensions, the cell pellet was resuspended with cold Carnoy's fixative (ethanol:acetic acid $=3: 1$ in volume). The suspension was placed on a glass microscope slide and the sample was dried at room temperature. The slides were treated with 2 M hydrochloric acid for 60 min at room temperature to dissociate the histones and partially denature the DNA. The slides were immersed in borax-borate buffer (pH 8.5) to neutralize the acid. BrdU-labeled tumor cells were detected by indirect immunofluorescence staining using a monoclonal anti-BrdU antibody (Becton Dickinson, San Jose, CA) and a fluorescein isothiocyanate (FITC)-conjugated antimouse IgG antibody (Sigma, St. Louis, MO). To observe the double staining of tumor cells with green-emitting FITC and red-emitting propidium iodide (PI), cells on the slides were treated with PI ( $2 \mu \mathrm{g} / \mathrm{ml}$ in PBS) and monitored under a fluorescence microscope.

The MN frequency in cells not labeled with BrdU could be examined by counting the micronuclei in the binuclear cells that showed only red fluorescence. The MN frequency was defined as the ratio of the number of micronuclei in the binuclear cells to the total number of binuclear 
cells observed.' The MN frequency has already been shown to be a tool for detecting radiosensitivity to carbon-ion beams. ${ }^{11}$

The ratios obtained in tumors not pretreated with BrdU indicated the MN frequency at all phases in the total tumor cell population. More than 400 binuclear cells were counted to determine the MN frequency.

\section{Clonogenic cell survival assay}

The clonogenic cell survival assay was also performed in the mice given no BrdU using an in vivo-in vitro assay method. Tumors were disaggregated by stirring for $20 \mathrm{~min}$ at $37^{\circ} \mathrm{C}$ in $\mathrm{PBS}$ containing $0.05 \%$ trypsin and $0.02 \%$ EDTA. The cell yield was $(4.5 \pm 1.1) \times 10^{7} / \mathrm{g}$ tumor weight.

As stated above, the MN frequencies for $Q$ cells were obtained from non-labeled tumor cells after continuous BrdU labeling. The MN frequencies and surviving fractions for the total cell population were obtained from cells in tumors not pretreated with BrdU. Thus, there was no effect of interaction between BrdU and irradiation on the values of MN frequency and SF. More than 3 tumor-bearing mice were used to assess each set of conditions and each experiment was repeated at least twice. To examine the differences between pairs of values, Student's t-test was used when variances of the two groups could be assumed to be equal; otherwise the Welch t-test was used. 
Influence of manipulating hypoxia on radiation dose-rate effect/Page 8

\section{Results}

The plating efficiency and MN frequency at 0 Gy are shown in Table 1. The plating efficiency was significantly smaller for the combination with MISO than for any other condition in the total cell population. In both the total and $Q$ cell populations, the MN frequency significantly increased in the following order: absolute control < totally hypoxic conditions < combination with nicotinamide < combination with misonidazole. Further, the MN frequency was significantly higher for Q cell population than the total cell population under all conditions.

Cell survival curves for the total tumor cells as a function of radiation dose after $\gamma$-ray irradiation are shown in Figure 1 . For both HDR and RDR irradiation, the surviving fractions (SFs) decreased in the following order: irradiation under totally hypoxic conditions $>$ aerobic irradiation without any drug > irradiation after nicotinamide loading $>$ irradiation after misonidazole loading. The SFs under all conditions increased as the dose rate of radiation decreased, especially after nicotinamide loading .

Cell survival curves for the total tumor cells as a function of radiation dose after accelerated carbon-ion beam irradiation with an LET of 18 and $50 \mathrm{keV} / \mu \mathrm{m}$ are shown in the left and right panels of Figure 2, respectively. For both HDR and RDR irradiation, the SFs decreased in the same order as for $\gamma$-ray irradiation, but the degree of change was reduced, especially with $50 \mathrm{keV} / \mu \mathrm{m}$ carbon-ion beams. The increases in the SF with the decrease in dose rate were suppressed compared with $\gamma$-ray irradiation, again especially with $50 \mathrm{keV} / \mu \mathrm{m}$ carbon-ion beams.

For baseline correction, we used the normalized MN frequency to exclude the MN frequency in non-irradiated tumors. The normalized MN 
Influence of manipulating hypoxia on radiation dose-rate effect/Page 9

frequency was the MN frequency in the irradiated tumors minus that in the non-irradiated tumors. Dose response curves of the normalized MN frequency for total and $Q$ tumor cell populations as a function of radiation dose after $\gamma$-ray irradiation are shown in the left and right panels of Figure 3, respectively. Overall, the normalized MN frequencies were significantly smaller in Q cells than the total cells. In both total and Q cells, under RDR as well as HDR irradiation, the normalized MN frequencies increased in the following order: irradiation under totally hypoxic conditions < aerobic irradiation without drugs < irradiation after nicotinamide loading < irradiation after misonidazole loading. The normalized MN frequencies decreased with the decrease in dose rate under all conditions, especially irradiation after nicotinamide loading in the total cell population.

Dose response curves of the normalized MN frequency for the total and $Q$ tumor cells as a function of radiation dose after accelerated carbon-ion beam irradiation with an LET of 18 (Figure 4) or $50 \mathrm{keV} / \mu \mathrm{m}$ (Figure 5) are shown in the left and right panels, respectively. Overall, the normalized MN frequencies were significantly smaller in Q cells than total cells, but the differences in radiosensitivity between the total and $Q$ cells were reduced compared with $\gamma$-ray irradiation, especially with $50 \mathrm{keV} /$ m carbon-ion beams. In both the total and Q cells, under RDR as well as HDR irradiation, the normalized MN frequencies increased in the same order as for $\gamma$-ray irradiation, but the degree of change was reduced, especially with $50 \mathrm{keV} / \mu \mathrm{m}$ carbon-ion beams. The decreases in the normalized MN frequency with the decrease in radiation dose rate were suppressed compared with $\gamma$-ray irradiation, again especially with $50 \mathrm{keV} / \mu \mathrm{m}$ carbon-ion beams. 
Influence of manipulating hypoxia on radiation dose-rate effect/Page 10

To estimate the effect of aerobic irradiation compared with hypoxic irradiation in both the total and $Q$ cells, the data for aerobic irradiation without any drug and irradiation under totally hypoxic conditions were used (Table 2). Following $\gamma$-ray irradiation, the values were significantly smaller for $Q$ cells than the total cells $(P<0.05)$, and in both the total and $Q$ cells, the values were significantly smaller for RDR than HDR irradiation $(P<0.05)$. In both populations, carbon-ion beams produced significantly smaller values than $\gamma$-rays $(P<0.05)$, and the values approached to 1.0 as the LET values increased.

To assess the radio-enhancing effect of nicotinamide or misonidazole under aerobic conditions in both the total and $Q$ cells compared with aerobic irradiation without any drug, the data for aerobic irradiation with and without drugs were used (Table 3). The enhancing effect of nicotinamide was more marked in the total cell population, especially with HDR $\gamma$-ray irradiation. The effect was little observed for RDR $\gamma$-ray irradiation and accelerated carbon-ion irradiation especially with a higher LET value. In contrast, the enhancing effect of misonidazole was more marked in the $Q$ cells. The enhancing effect was also attenuated for accelerated carbon-ion irradiation especially with a higher LET value.

To investigate the reduction in radiosensitivity caused by a decrease in radiation dose rate, dose-modifying factors were calculated using the data for all irradiation conditions given in Figures 1 through 5 (Table 4). On the whole, the reduction in radiosensitivity was more marked in $Q$ than the total cells, especially under $\gamma$-ray irradiation. In the total cells, the degree of the reduction of radiosensitivity was reduced in the following order: aerobic irradiation with nicotinamide 
Influence of manipulating hypoxia on radiation dose-rate effect/Page 11

\begin{abstract}
$>$ aerobic irradiation without any drug > aerobic irradiation with misonidazole > irradiation under totally hypoxic conditions. In Q cells, the degree of the reduction of radiosensitivity was reduced in the following order: aerobic irradiation with nicotinamide = aerobic irradiation without any drug > irradiation under totally hypoxic conditions $>$ aerobic irradiation with misonidazole. This order of the reduction in radiosensitivity and the difference in the reduction in radiosensitivity between $Q$ and the total cells became more indistinct with the use of accelerated carbon-ion beams, especially at a higher LET value, than with the use of $\gamma$-rays.
\end{abstract}

To examine the difference in radiosensitivity between the total and $Q$ cells, dose-modifying factors, which allow us to compare the dose of radiation necessary to obtain a normalized MN frequency of 0.2 in $Q$ cells with that in the total cells, were calculated using the data in Figures 2 and 3 (Table 5). Overall, the difference in radiosensitivity was greater under RDR than HDR irradiation, especially with $\gamma$-rays. The difference in radiosensitivity increased in the following order: irradiation under totally hypoxic conditions < aerobic irradiation with misonidazole < aerobic irradiation without any drug $\leq$ aerobic irradiation with nicotinamide. This order of the increase in the difference in radiosensitivity and the difference itself in radiosensitivity became more indistinct with the use of accelerated carbon-ion beams, especially at a higher LET value, than with the use of $\gamma$-rays. 
Influence of manipulating hypoxia on radiation dose-rate effect/Page 12

\section{Discussion}

Solid tumors, especially human tumors, are thought to contain a high proportion of $Q$ cells. ${ }^{6}$ The presence of $Q$ cell is probably due, in part, to hypoxia and the depletion of nutrients in the tumor core, another consequence of poor vascular supply. ${ }^{6}$ This might promote the formation of micronuclei at 0 Gy in Q tumor cells (Table 1 ). Q cells were shown to have significantly less radiosensitivity than the total cells here (Figs. 3 through 5 ). This means that more Q cells survive radiation therapy than $\mathrm{P}$ cells. Thus, the control of $Q$ cells has a great impact on the outcome of radiation therapy. In both the total and $Q$ cell populations, carbon ion irradiation was less dependent on oxygenation status with little recovery from radiation-induced DNA damage, leading to high RBE values compared with $\gamma$-ray irradiation. ${ }^{11}$ In terms of the tumor cell-killing effect as a whole, including intratumor Q cell control, carbon-ion beam radiotherapy can be a promising treatment for refractory tumors.

Q cell population had been shown to include a significantly larger hypoxic fraction than the total cell population, ${ }^{12}$ resulting in a significantly smaller effect of carbon-ion beams under aerobic irradiation compared with hypoxic irradiation in Q cells (Table 2 ). Since tumor radiosensitivity was significantly less dependent on intratumor oxygenation status and the irradiation dose rate effect when carbon-ion beams, especially with a higher LET value, were used, a much smaller effect of aerobic irradiation compared with hypoxic irradiation was observed here. This was partly because the frequency of closely spaced DNA lesions forming a cluster of DNA damage produced by high LET carbon-ion irradiation is much less dependent on oxygenation status 
Influence of manipulating hypoxia on radiation dose-rate effect/Page 13

at the time of irradiation than that of DNA damage produced by low LET $\gamma$-ray irradiation..$^{13}$

Tumor hypoxia is a direct consequence of structural abnormalities of the microvasculature and functional abnormalities of the microcirculation in solid tumors and results from either limited oxygen diffusion (chronic hypoxia) or limited perfusion (acute hypoxia, transient hypoxia, or ischemic hypoxia). Large intercapillary distances resulting from rapid tumor cell proliferation lead to chronically hypoxic cells existing at the rim of the oxygen diffusion distance. ${ }^{14}$ Factors such as vessel plugging by blood cells or circulating tumor cells, the collapse of vessels in regions of high tumor interstitial pressure, or spontaneous vasomotor activity in normal tissue vessels incorporated into the tumor which subsequently affects flow in downstream tumor microvessels cause intermittent blood flow in tumors, which results in acute hypoxia. ${ }^{15}$ Thus, acute hypoxic areas are distributed throughout the tumor depending on these causative factors and can occur sporadically in large areas of a solid tumor. Nicotinamide, a vitamin $B_{3}$ analogue, is known to prevent these transient fluctuations in tumor blood flow that lead to the development of acute hypoxia. ${ }^{9}$ Misonidazole is a typical 2-nitro-imidazole hypoxic cell sensitizer which is thought to function as an oxygen-mimicking agent in intratumor hypoxic areas under irradiation. ${ }^{10}$ In SCC VII tumors, it had been shown that the hypoxic fraction of the total cell population is predominantly made up of acute hypoxic areas and that of the hypoxia rich-Q cell population is mainly made up of chronic hypoxic areas. ${ }^{12}$ Therefore, under HDR $\gamma$-ray irradiation, the enhancement ratio of nicotinamide was higher in the total cells than in Q cells, and that of misonidazole was higher 
Influence of manipulating hypoxia on radiation dose-rate effect/Page 14

in the Q cells (Table 3 ). When carbon-ion beams, especially with a higher LET value, were employed, these differences in the enhancement ratio were reduced because radiosensitivity was much less dependent on intratumor oxygenation status. ${ }^{11}$ However, under RDR $\gamma$-ray irradiation, acute hypoxic areas appear and disappear throughout a solid tumor during long periods of irradiation. ${ }^{15}$ As a result, RDR irradiation even without nicotinamide could make it possible to irradiate all acute hypoxic areas under oxic conditions, leading to no radio-enhancing effect of nicotinamide. The radio-enhancing effect of misonidazole, which depends on the size of the hypoxic fraction in solid tumors, however, still could be observed under RDR as well as HDR irradiation.

Enhancement of the irradiation dose rate effect on the normalized frequency of micronuclei by $\gamma$-ray irradiation in the presence of nicotinamide compared with $\gamma$-ray irradiation alone was observed in the acute hypoxia-rich total cells rather than the chronic hypoxia-rich Q cells (Table 4). Suppression of the dose rate effect by inducing total hypoxia during irradiation was slightly more clearly observed in normoxia-rich total cell than hypoxia-rich Q cells. Some recent studies in vitro found that hypoxia-induced translational repression can explain the decreased homologous recombination (HR) repair of radiation-induced DNA double-stranded breaks (dsbs) ${ }^{16}$ a more important mechanism for the repair of dsbs in late-S and G2, and that HR plays a greater role in determining hypoxic radiosensitivity than normoxic radiosensitivity. ${ }^{17}$ Thus, the use of nicotinamide or tumor clamping to induce total hypoxia influenced repair more in the total cells including late-S and G2 phase cells than in Q cells. In contrast, the repression of the dose rate effect by the hypoxic cell radio-sensitizer 
Influence of manipulating hypoxia on radiation dose-rate effect/Page 15

misonidazole was slightly more clearly observed in the hypoxia-rich $Q$ cells than normoxia-rich total cells. It had already been shown that the loading of misonidazole after low LET HDR irradiation to solid tumors inhibited the recovery from radiation-induced PLD in vivo, especially in $Q$ cells. ${ }^{18}$ In this study, it was shown that misonidazole combined with low LET RDR irradiation could suppress the dose rate effect, especially in Q cells. According to the previous finding that the recovery from PLD and the decrease in radiosensitivity through a reduction in the irradiation dose rate under low LET irradiation are mainly due to non-homologous end-joining (NHEJ) repair, which is the predominant DNA dsbs repair process for cells in G0, G1 or early-S phase, ${ }^{19}$ misonidazole itself or protein adducts of reductively-activated misonidazole in hypoxic areas in irradiated tumors may inhibit NHEJ more efficiently than HR. ${ }^{10}$ Again, when carbon-ion beams, especially with a higher LET value, were employed, the effects of combined treatment on the irradiation dose rate effect became indistinct because radiosensitivity was much less dependent on intratumor oxygenation status and the irradiation dose rate. ${ }^{11}$

Overall, the difference in radiosensitivity between the total and Q cells was increased by reducing the dose rate, especially for $\gamma$-ray irradiation, because of the greater reduction in radiosensitivity caused by a decrease in the dose rate in $Q$ cells than in the total cells ${ }^{9}$ (Table 5). Nicotinamide enhanced the radiosensitivity of the total cells, leading to a widening of the difference in radiosensitivity between the total and Q cell populations compared with aerobic HDR $\gamma$-ray irradiation without any drug. But, for RDR $\gamma$-ray irradiation, the effect of nicotinamide disappeared due to the characteristics of acute hypoxia 
Influence of manipulating hypoxia on radiation dose-rate effect/Page 16

in solid tumors, resulting in no change in the difference in radiosensitivity between the total and Q cells compared with $\gamma$-ray irradiation only. Meanwhile, under totally hypoxic conditions, the difference in radiosensitivity was smaller than for aerobic $\gamma$-ray irradiation because of the radio-resistance induced by total hypoxia in both the total and $Q$ cell populations in sold tumors. Misonidazole enhanced the radiosensitivity of the hypoxia-rich $Q$ cells much more than that of the total cells under both HDR and RDR $\gamma$-ray irradiation, leading to a decrease in the difference in radiosensitivity between the two cells compared with aerobic $\gamma$-ray irradiation only. When carbon-ion beams, especially with a higher LET value, were employed, the effects of combined treatment on the difference in radiosensitivity between the total and $Q$ cells became indistinct because tumor radiosensitivity was much less dependent on intratumor oxygenation status and the irradiation dose rate. ${ }^{11}$ Anyway, at least in this study, it was elucidated that manipulating hypoxia during RDR irradiation, especially with $\gamma$-rays, influences tumor radiosensitivity as well as HDR irradiation in both total and $Q$ cell populations. In radiotherapy, irradiation dose rate also should be taken into account when intratumor hypoxia is manipulated. 


\section{References}

1. Ahmed RS, Kim RY, Duan J, Meleth S, De Los Santos JF, Fiveash JB. IMRT dose escalation for positive para-aortic lymph nodes in patients with locally advanced cervical cancer while reducing dose to bone marrow and other organs at risk. Int J Radiat Oncol Biol Phys $2005 ; 60: 505-512$.

2. Wulf J, Haedinger U, Oppitz U, Thiele W, Mueller G, Flentje M. Stereotactic radiotherapy for primary lung cancer and pulmonary metastases: a noninvasive treatment approach in medically inoperable patients. Int J Radiat Oncol Biol Phys 2004;60:186-196.

3. Hall EL, Giaccia AJ. Repair of radiation damage and the dose-rate effect. In: Hall EJ, Giaccia AJ, editors. Radiobiology for the Radiologist, 6th ed. Philadelphia, Lippincott Williams \& Wilkins; 2006 p 60-84.

4. Hall EL, Giaccia AJ. Linear energy transfer and relative biological effectiveness. In: Hall EJ, Giaccia AJ, editors. Radiobiology for the Radiologist, 6th ed. Philadelphia, Lippincott Williams \& Wilkins; 2006 p 106-116.

5. Hall EL, Giaccia AJ. Oxygen effect and reoxygenation. In: Hall EJ, Giaccia AJ, editors. Radiobiology for the Radiologist, 6 th ed. Philadelphia, Lippincott Williams \& Wilkins; 2006 p 85-105.

6. Vaupel P. Tumor microenvironmental physiology and its implications for radiation oncology. Semin Radiat Oncol 2004;14:197-275.

7. Masunaga S, Ono K. Significance of the response of quiescent cell populations within solid tumors in cancer therapy. J Radiat Res $2002 ; 43: 11-25$.

8. Torikoshi M, Minohara S, Kanematsu N, Komori M, Kanazawa M, Noda 
Influence of manipulating hypoxia on radiation dose-rate effect/Page 18

K, et al. Irradiation system for HIMAC. J Radiat Res $2007 ; 48($ Suppl) :A15-A25.

9. Chaplin DJ, Horsman MR, Trotter MJ. Effect of nicotinamide on the microregional heterogeneity of oxygen delivery within a murine tumor. J Natl Cancer Inst 1990;82:672-676.

10. Wardman P. Chemical radiosensitizers for use in radiotherapy. Clin Oncol 2007;19:397-417.

11. Masunaga S, Ando K, Uzawa A, Hirayama R, Furusawa Y, Sakurai Y, et al. Radiobiologic significance of the response of intratumor quiescent cells in vivo to accelerated carbon ion beams compared with $\gamma$-rays and reactor neutron beams. Int J Radiat Oncol Biol Phys $2008 ; 70: 221-228$.

12. Masunaga S, Ono K, Suzuki M, Nishimura Y, Hiraoka M, Kinashi Y, et al. Alteration of the hypoxic fraction of quiescent cell populations by hyperthermia at mild temperatures. Int J Hyperthermia $1997 ; 13: 401-411$.

13. Hada M, Georgakilas AG. Formation of clustered DNA damage after high-LET irradiation: a Review. J Radiat Res 2008;49:203-210.

14. Thomlinson RH, Gray LH. The histological structure of some human lung cancers and the possible implications for radiotherapy. Br J Cancer 1955;9:539-549.

15. Brown JM. Evidence of acutely hypoxic cells in mouse tumours, and a possible mechanism of reoxygenation. Br J Radiol 1979;52:650-656.

16. Chan N, Koritzinsky M, Zhao H, Bindra R, Glazer PM, Powell S, et al. Chronic hypoxia decreases synthesis of homologous recombination proteins to offset chemoresistance and radioresistance. Cancer Res $2008 ; 68: 605-614$. 
17. Sprong D, Janssen HL, Vens C, Begg AC. Resistance of hypoxic cells to ionizing radiation is influenced by homologous recombination status. Int J Radiat Oncol Biol Phys 2006;64:562-572.

18. Masunaga S, Ono K, Abe M. Potentially lethal damage repair by quiescent cells in murine solid tumors. Int J Radiat Oncol Biol Phys $1992 ; 22: 973-978$.

19. Masunaga S, Nagata K, Suzuki M, Kashino G, Kinashi Y, Ono K. Inhibition of repair from radiation-induced damage by mild temperature hyperthermia, referring to the effect on quiescent cell populations. Radiat Med 2007;25:417-425. 


\section{Captions for Illustrations}

Fig. 1. Cell survival curves for the total tumor cell population as a function of radiation dose after $\gamma$-ray irradiation. Open and solid symbols represent the surviving fractions after high dose-rate and reduced dose-rate $\gamma$-ray irradiation, respectively. Circles, reversed triangles, triangles, and squares represent the surviving fractions after aerobic irradiation without any drug, irradiation under totally hypoxic conditions, aerobic irradiation after nicotinamide (NA) loading, and aerobic irradiation after misonidazole (MISO) loading, respectively. Bars represent standard errors.

Fig. 2. Cell survival curves for the total tumor cell population as a function of radiation dose after accelerated carbon-ion beam irradiation with an LET of 18 and $50 \mathrm{keV} / \mu \mathrm{m}$ are shown in the left and right panels, respectively. Open and solid symbols represent the surviving fractions after high dose-rate and reduced dose-rate accelerated carbon-ion beam irradiation, respectively. Circles, reversed triangles, triangles, and squares represent the surviving fractions after aerobic irradiation without any drug, irradiation under totally hypoxic conditions, aerobic irradiation after nicotinamide (NA) loading, and aerobic irradiation after misonidazole (MISO) loading, respectively. Bars represent standard errors.

Fig. 3. Dose response curves of the normalized micronucleus frequency for the total and quiescent tumor cell populations as a function of radiation dose after $\gamma$-ray irradiation are shown in the left and right panels, respectively. Open and solid 
Influence of manipulating hypoxia on radiation dose-rate effect/Page 21

symbols represent the normalized micronucleus frequencies after high dose-rate and reduced dose-rate $\gamma$-ray irradiation, respectively. Circles, reversed triangles, triangles, and squares represent the normalized micronucleus frequencies after aerobic irradiation without any drug, irradiation under totally hypoxic conditions, aerobic irradiation after nicotinamide (NA) loading, and aerobic irradiation after misonidazole (MISO) loading, respectively. Bars represent standard errors.

Fig. 4. Dose response curves of the normalized micronucleus frequency for the total and quiescent tumor cell populations as a function of radiation dose after accelerated carbon-ion beam irradiation with an LET of $18 \mathrm{keV} / \mu \mathrm{m}$ are shown in the left and right panels, respectively. Open and solid symbols represent the normalized micronucleus frequencies after high dose-rate and reduced dose-rate accelerated carbon-ion beam irradiation, respectively. Circles, reversed triangles, triangles, and squares represent the normalized micronucleus frequencies after aerobic irradiation without any drug, irradiation under totally hypoxic conditions, aerobic irradiation after nicotinamide (NA) loading, and aerobic irradiation after misonidazole (MISO) loading, respectively. Bars represent standard errors.

Fig. 5. Dose response curves of the normalized micronucleus frequency for the total and quiescent tumor cell populations as a function of radiation dose after accelerated carbon-ion beam irradiation with an LET of $50 \mathrm{keV} / \mu \mathrm{m}$ are shown in the left and 
right panels, respectively. Open and solid symbols represent the normalized micronucleus frequencies after high dose-rate and reduced dose-rate accelerated carbon-ion beam irradiation, respectively. Circles, reversed triangles, triangles, and squares represent the normalized micronucleus frequencies after aerobic irradiation without any drug, irradiation under totally hypoxic conditions, aerobic irradiation after nicotinamide (NA) loading, and aerobic irradiation after misonidazole (MISO) loading, respectively. Bars represent standard errors. 
Table 1.

Plating efficiency and micronucleus frequency at 0 Gy

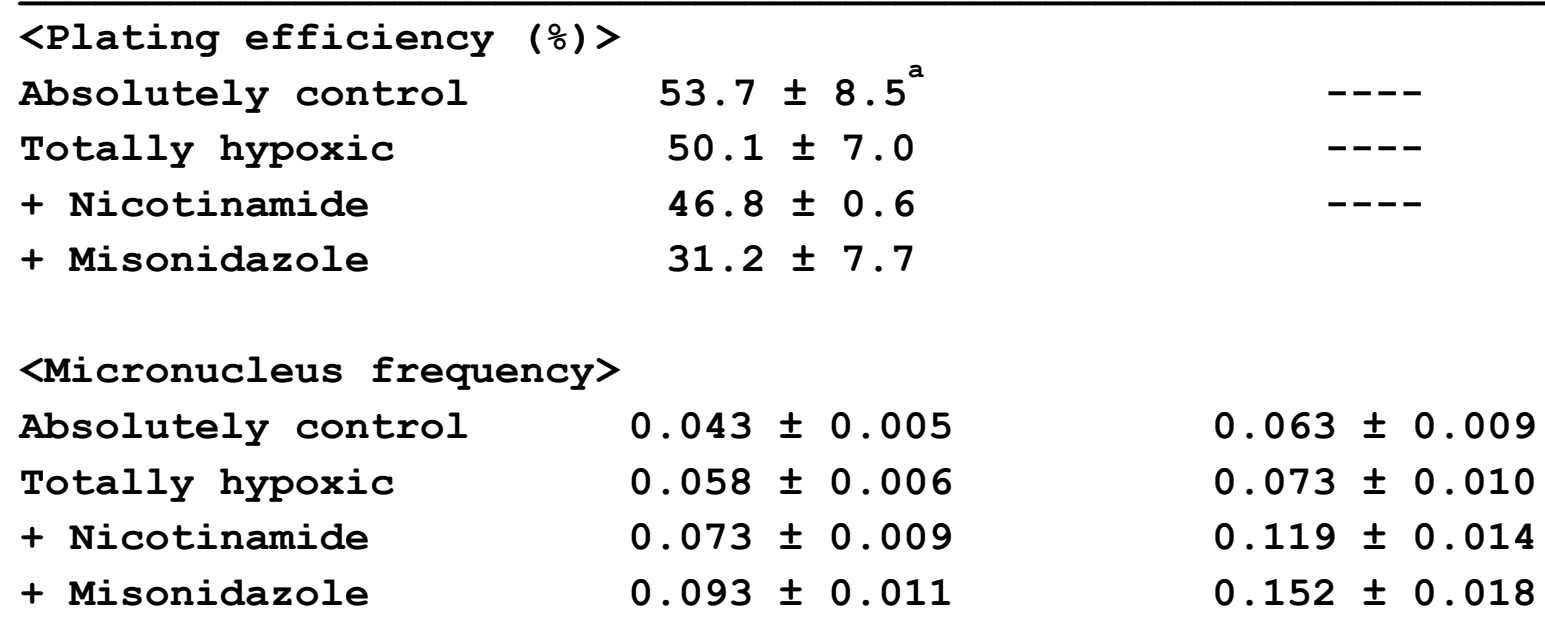


Table 2.

Irradiation under aerobic conditions compared with irradiation under hypoxic conditions ${ }^{a}$

\begin{tabular}{ccc}
\hline \hline -Rays & Carbon-ion beams & Carbon-ion beams \\
& $(18 \mathrm{keV} / \mu \mathrm{m})$ & $(50 \mathrm{keV} / \mu \mathrm{m})$
\end{tabular}

<Surviving fraction $=0.3>$

Total cells

High dose-rate irradiation
$2.1(2.0-2.2)$
$1.4(1.3-1.5)$
$1.15(1.1-1.2)$

Reduced dose-rate irradiation
$1.8(1.7-2.0)$
$1.25(1.15-1.35)$
$1.1(1.05-1.15)$

<Normalized micronucleus frequency $=0.2>$

Total cells

High dose-rate irradiation
$1.9(1.8-2.0)$
$1.35(1.25-1.45)$
$1.2(1.1-1.3)$

Reduced dose-rate irradiation
$1.7(1.6-1.8)$
$1.25(1.15-1.35)$
$1.15(1.1-1.2)$

Quiescent cells

High dose-rate irradiation
$1.5(1.4-1.6)$
$1.25(1.15-1.35)$
$1.15(1.1-1.2)$

Reduced dose-rate irradiation
$1.3(1.2-1.4)$
$1.1(1.05-1.15)$
$1.05(1.0-1.1)$

$\overline{\text {; The ratio of the dose of radiation necessary to obtain each end-point }}$ under hypoxic conditions to that needed to obtain each end-point under

b aerobic conditions.

; Values in parentheses are 95\% confidence limits, determined using standard errors. If the ranges of $95 \%$ confidence limits showed no overlap between any two values, the difference between the two values was considered significant $(p<0.05)$. 
Table 3.

Enhancement ratios ${ }^{a}$ due to combination with nicotinamide or misonidazole

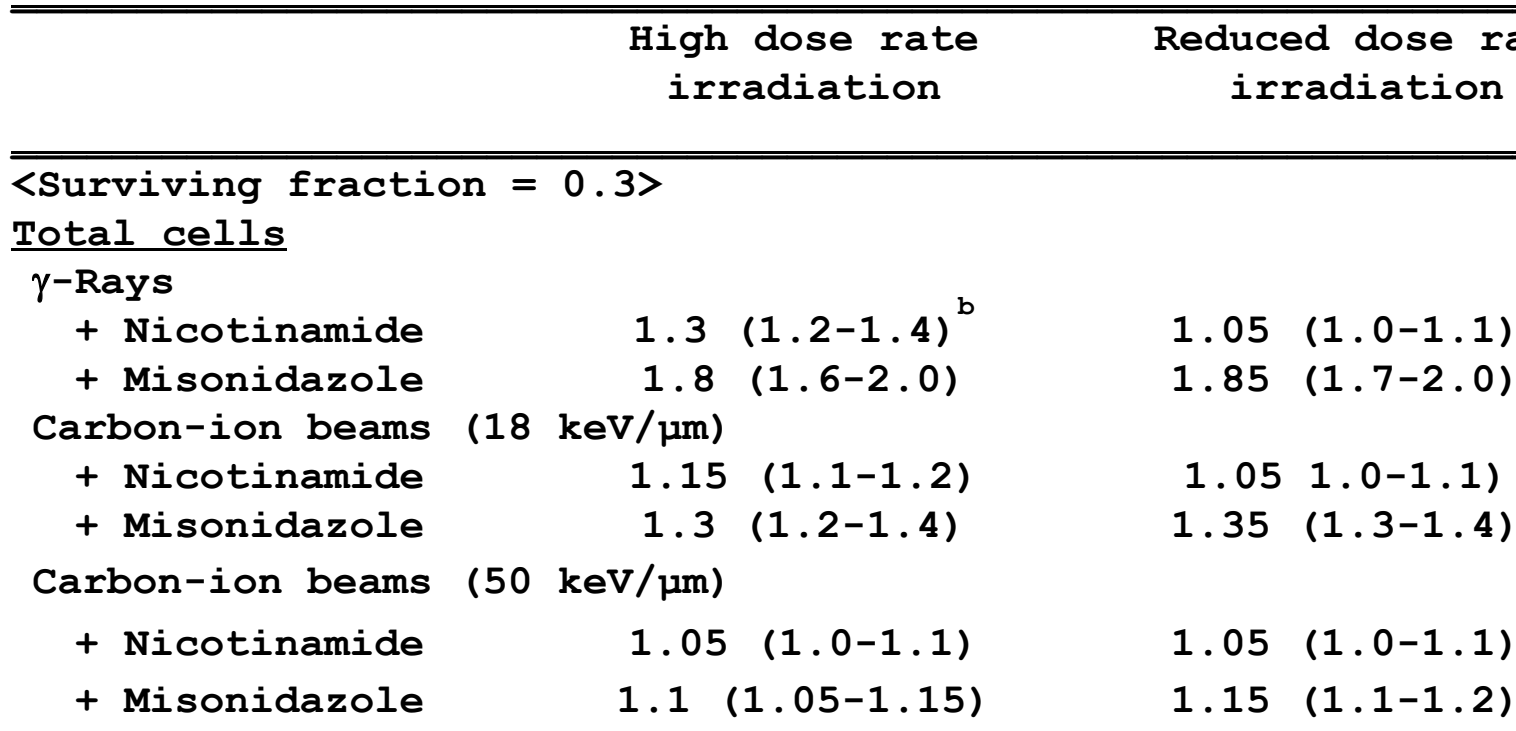

<Normalized micronucleus frequency $=0.2>$

Total cells

$\gamma$-Rays

+ Nicotinamide

+ Misonidazole

Carbon-ion beams

+ Nicotinamide

(18 $\mathrm{keV} / \mathrm{\mu m})$

$1.2(1.1-1.3)$

$1.05(1.0-1.1)$

+ Misonidazole

$1.15(1.1-1.2)$

$1.35(1.25-1.45)$

Carbon-ion beams

+ Nicotinamide

1.2 (1.1-1.3)

1.05 (1.0-1.1)

(50 kev/ $/ \mathrm{m})$

+ Misonidazole

1.05 (1.0-1.1)

$1.25(1.15-1.35)$

Quiescent cells

$$
\begin{aligned}
& \gamma \text {-Rays } \\
& + \text { Nicotinamide } \\
& + \text { Misonidazole }
\end{aligned}
$$

Carbon-ion beams

+ Nicotinamide

$(18 \mathrm{keV} / \mu \mathrm{m})$

1.1 (1.05-1.15)

1.45 (1.35-1.55)

+ Misonidazole

Carbon-ion beams

+ Nicotinamide

(50 keV/ $\mathrm{mm}$ )
$1.05 \quad(1.0-1.1)$

$1.05(1.0-1.1)$
$1.05(1.0-1.1)$

1.1 (1.05-1.15)
$1.05(1.0-1.1)$

1.5 (1.4-1.6)

$1.0(0.95-1.05)$

$1.3(1.2-1.4)$

$1.0(0.95-1.05)$

$1.1(1.05-1.15)$

\footnotetext{
; The ratio of the dose of radiation necessary to obtain each end-point without the drug to that needed to obtain each end-point with the drug.

; As in Table 2.
} 
Table 4.

Dose-modifying factors due to the reduction in radiosensitivity caused by a decrease in radiation dose rate

\begin{tabular}{ccc}
\hline \hline$\gamma$-Rays & Carbon-ion beams & Carbon-ion beams \\
& $(18 \mathrm{keV} / \mu \mathrm{m})$ & $(50 \mathrm{keV} / \mu \mathrm{m})$
\end{tabular}

<Surviving fraction $=0.3>$

Total cells

Radiation only
$1.3(1.2-1.4)^{\mathrm{b}}$
$1.2(1.1-1.3)$
$1.15(1.1-1.2)$

Totally hypoxic
$1.2(1.1-1.3)$
$1.15(1.05-1.25)$
$1.1(1.05-1.15)$

+ Nicotinamide
$1.55(1.4-1.7)$
$1.35(1.25-1.45)$
$1.2(1.15-1.25)$

+ Misonidazole
$1.25(1.15-1.35)$
$1.2(1.1-1.3)$
$1.15(1.1-1.2)$

<Normalized micronucleus frequency $=0.2>$

Total cells

Radiation only
$1.25(1.15-1.35)$

$$
1.2(1.1-1.3)
$$
$1.1(1.05-1.15)$

Totally hypoxic

$1.1(1.05-1.15)$

$1.05(1.0-1.1)$

+ Nicotinamide

$$
1.4(1.25-1.55) \quad 1.3(1.2-1.4) \quad 1.2(1.1-1.3)
$$

+ Misonidazole
$1.2(1.1-1.3)$
$1.15(1.05-1.25)$
$1.1(1.05-1.15)$

Quiescent cells

Radiation only
$1.4(1.3-1.5)$
$1.3(1.2-1.4)$
$1.25(1.15-1.35)$

Totally hypoxic

$1.15(1.1-1.2) \quad 1.1(1.05-1.15)$

+ Nicotinamide

$1.25(1.15-1.35) \quad 1.15(1.1-1.2) \quad 1.1(1.05-1.15)$

1.4 (1.3-1.5)

$1.3(1.2-1.4)$

$1.25(1.15-1.35)$

+ Misonidazole
1.15 (1.1-1.2)
$1.1(1.05-1.15)$
1.1 (1.05-1.15)

\section{; As in Table 2 .}


Table 5 .

Dose-modifying factors for quiescent cells relative to total tumor cells

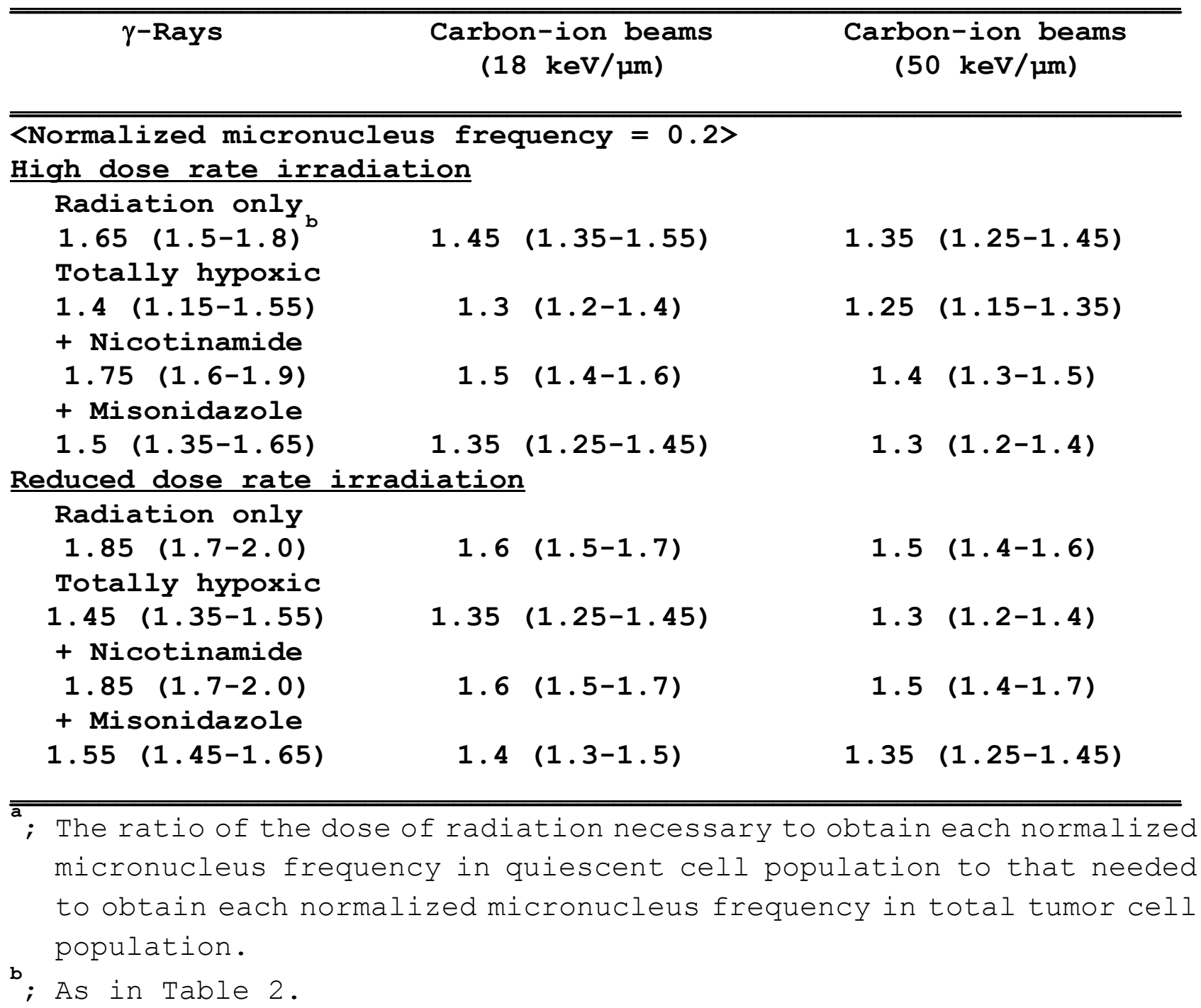




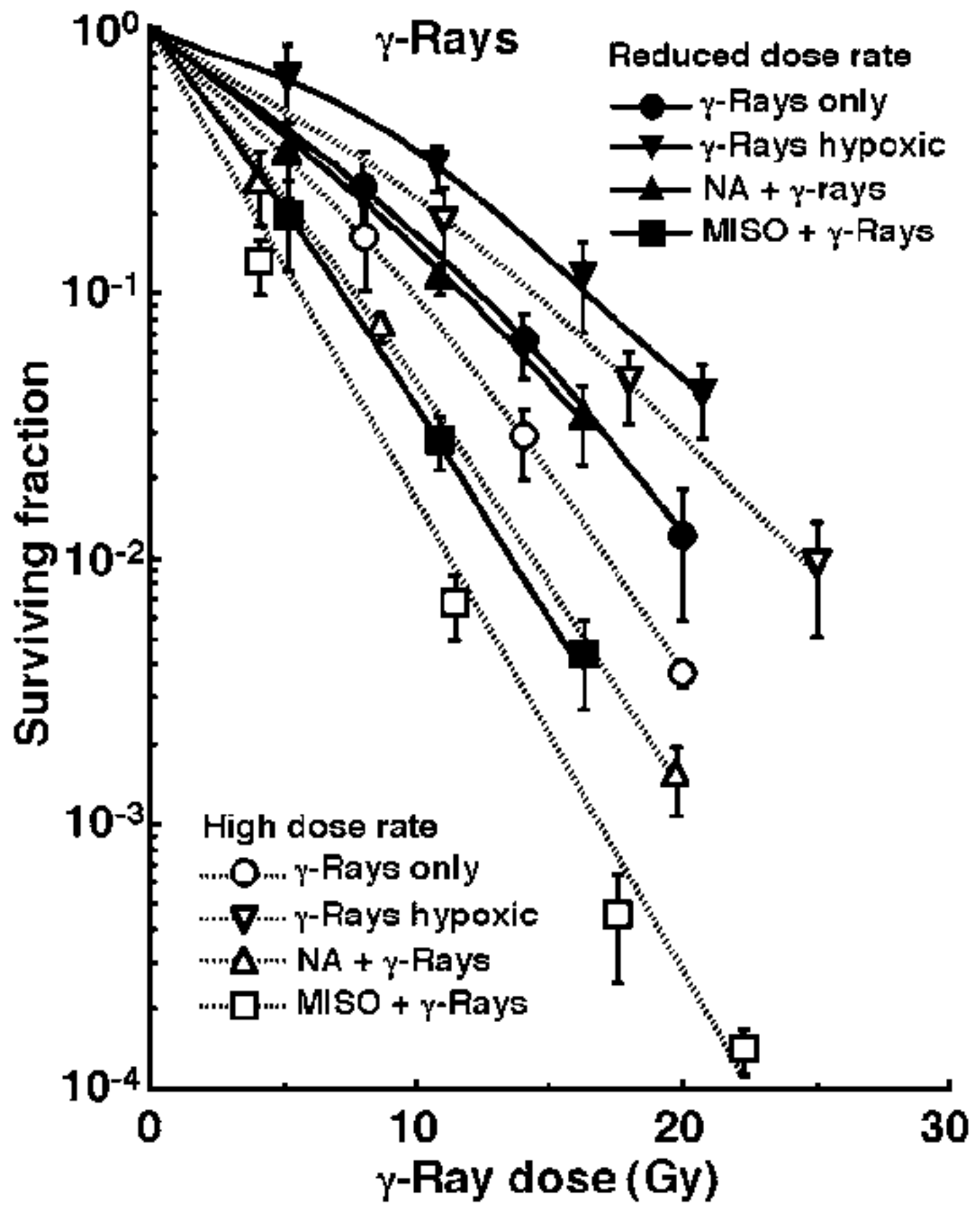




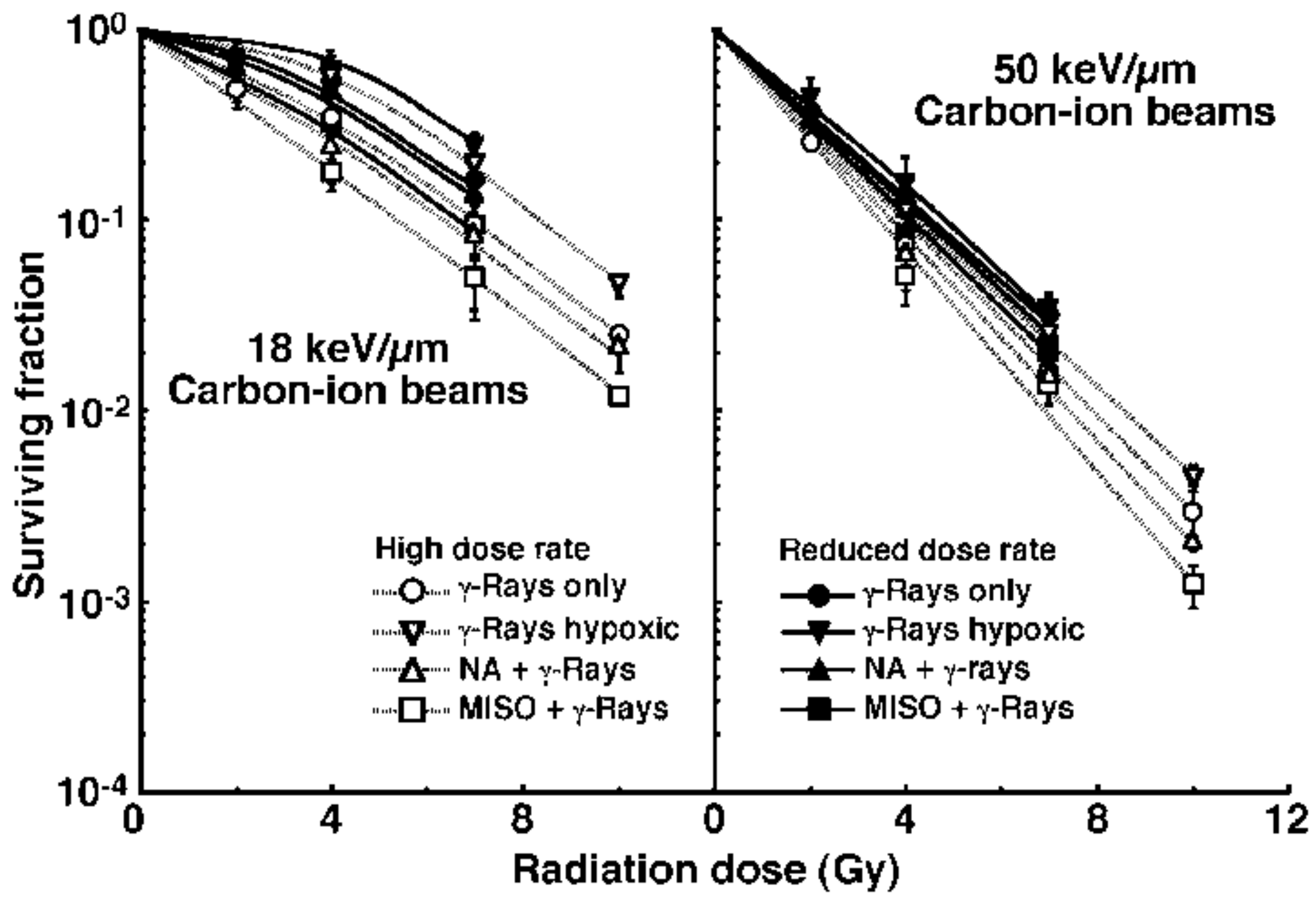




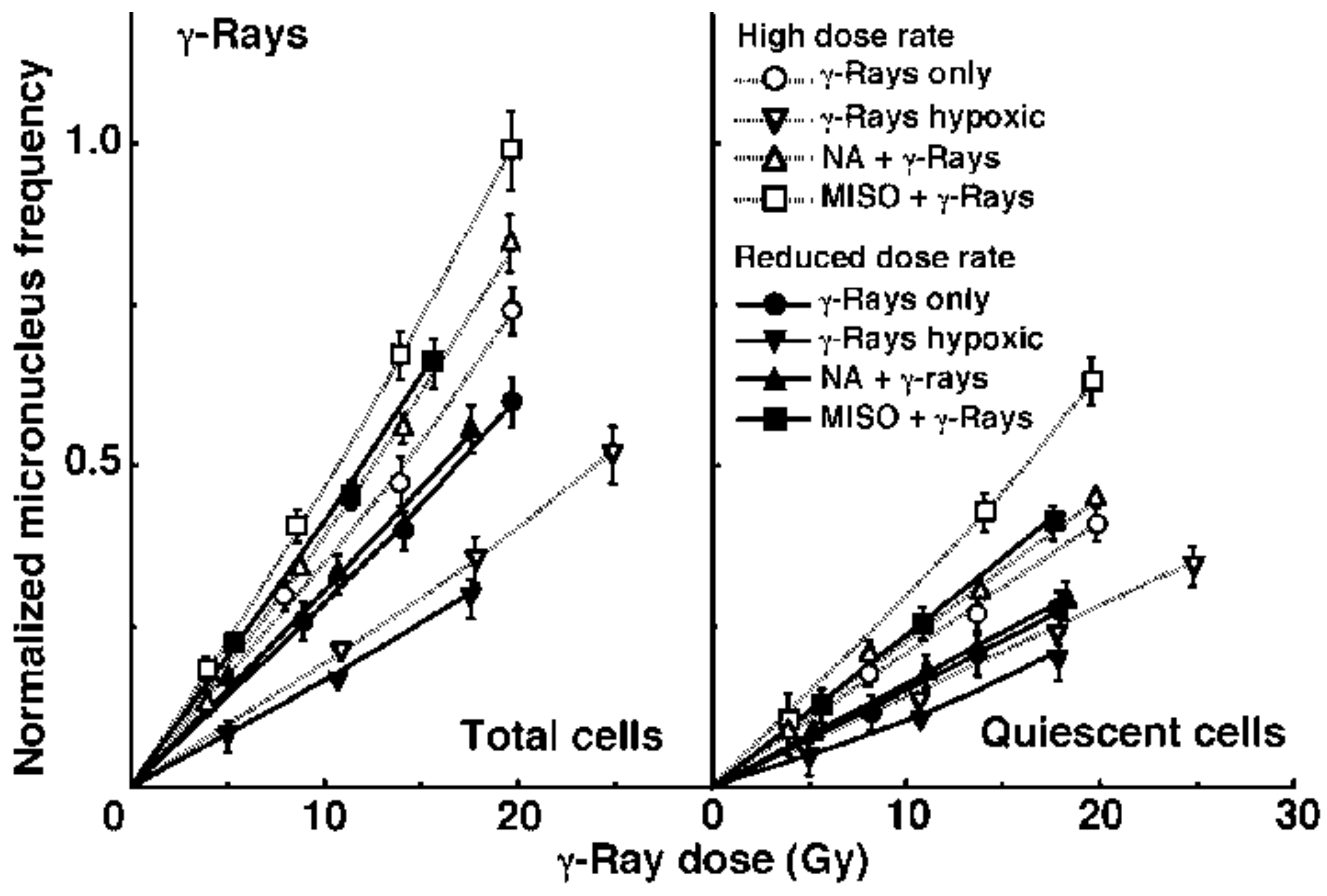




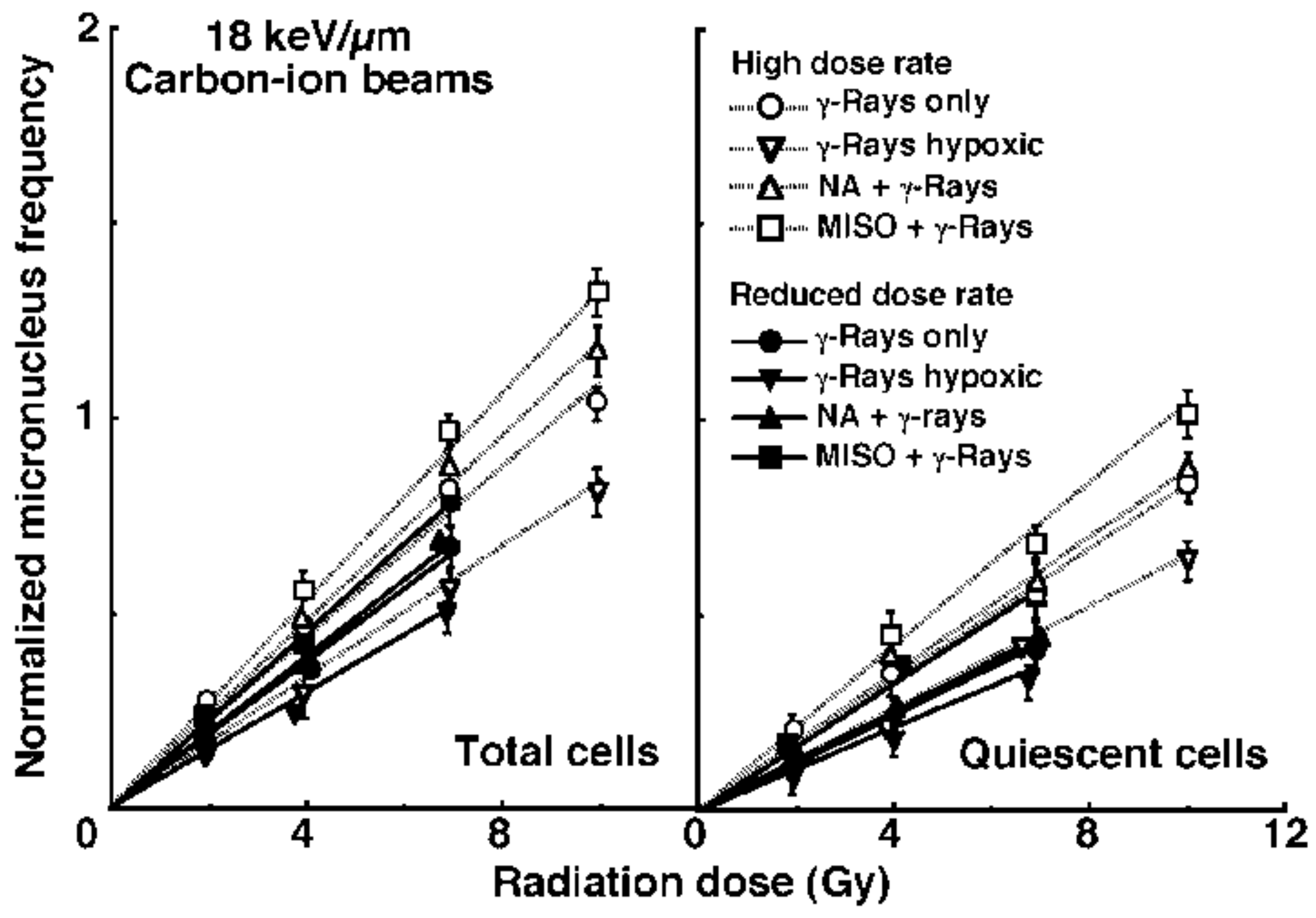




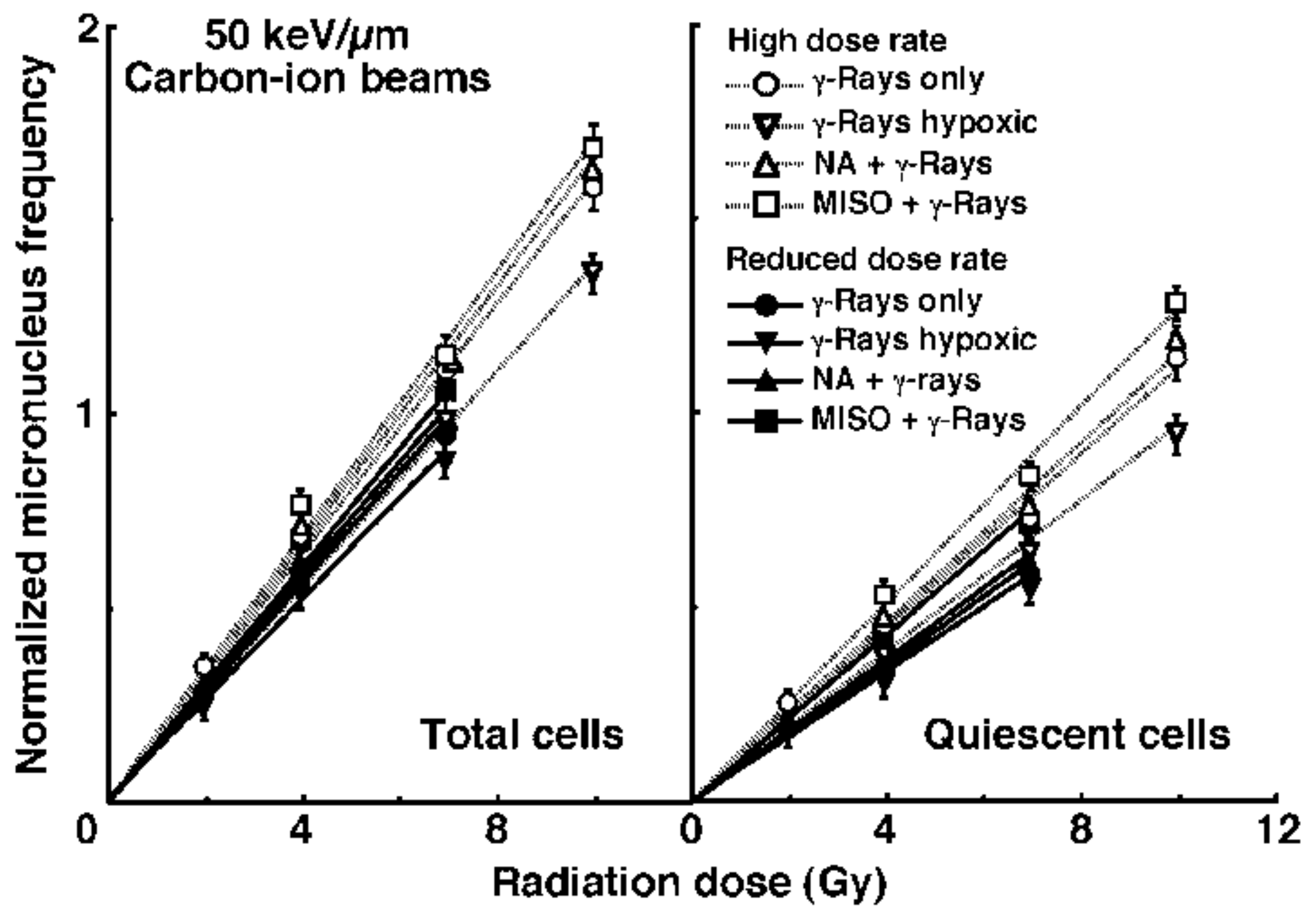




\section{Acknowledgments}

This study was supported, in part, by a Grant-in-aid for Scientific Research (C) (20591493) from the Japan Society for the Promotion of Science. 